\title{
Thermal abundance of hyperons from a coupled channel model
}

\author{
Pok Man $\mathrm{Lo}^{1, *}$ \\ ${ }^{1}$ Institute of Theoretical Physics, University of Wroclaw, PL-50204 Wrocław, Poland
}

\begin{abstract}
I demonstrate how the thermal abundances of $S=-1$ strange baryons can be computed based on the density of states extracted from a coupled-channel model.
\end{abstract}

\section{Introduction}

The S-matrix formulation of statistical mechanics $[1,2]$ offers a unique theoretical framework to examine how interactions among constituents could dictate the bulk properties of the medium. The essential connection is provided by the density of states (DoS) [3, 4], which can be expressed in terms of the S-matrix by

$$
\begin{aligned}
B(E) & =\frac{1}{2} \operatorname{Im} \operatorname{Tr}\left[S^{-1} \frac{d}{d E} S-\left(\frac{d}{d E} S^{-1}\right) S\right] \\
& =\frac{d}{d E} \operatorname{Im} \ln \operatorname{det} S(E) .
\end{aligned}
$$

The thermal partition function is given by an integral of the DoS with the appropriate Boltzmann weight. For example the thermal abundance due to the interaction can be computed as

$$
\Delta n_{a}=\int \frac{d E}{2 \pi} B(E) n_{a}^{(0)}(E, T)
$$

where $n_{a}^{(0)}\left(E=m_{a}, T\right)$ denotes the particle density of an ideal gas of particles with mass $m_{a}$. Other thermal (and fluctuation) observables can be analogously computed [5,6].

Writing the DoS in terms of the S-matrix makes possible to incorporate precise hadron properties, e.g. spectra, production mechanisms and decays, obtained from analyzing volumes of experimental data with different theoretical approaches, into the construction of thermal model. The S-matrix language also naturally describes coupled-channels effects: overlapping resonances, inelasticity, and various threshold effects as channels open up. These phenomena of hadron physics, observed in most known resonances, go beyond what can be captured with the compilation of an appropriate list of resonances, or by introducing some general repulsions among constituents. In this regard, nature is far more creative.

\footnotetext{
*e-mail: pokman.lo@uwr.edu.pl
} 
Of course, determining the full $\mathrm{N}$-body, multi-channel S-matrix of an interacting system is in general very difficult (if not impossible). This is reflected, e.g., in the poorly constrained couplings and / or the various background channels introduced to satisfy unitarity. The positive aspect is that such model would be systematically improved with data from further scattering experiments. For now we shall take these limitations as features of the approach and explore the perspectives they give when applied to studying thermal system.

\section{DoS of coupled-channel hyperon system}

A coupled-channel approach [7] is mandatory to describe the $S=-1$ hyperon system: the lowest channels open up in a similar energy range, and most resonances decay into multiple channel. To tackle a thermal system, prescription (1) remains valid, but the S-matrix should be formally understood as a matrix acting in the open-channel space, i.e. an $N_{\text {chan. }} \times N_{\text {chan. }}$. matrix. See table 1 for the list of channels considered.

The trace operation (Tr) in Eq. (1), originated from the thermal trace in constructing the partition function, enforces a summation over the $N_{\text {chan. }}$ channels. The effective spectral function describing a multichannel system reads $[4,6,8]$

$$
B(M)=\sum_{a=1}^{N_{\text {chan. }}} B_{a}(M),
$$

where

$$
B_{a}(M)=\frac{1}{2} \operatorname{Im}\left[S^{-1} \frac{d}{d M} S-\left(\frac{d}{d M} S^{-1}\right) S\right]_{a a} .
$$

Here $[\ldots]_{a a}$ means the $a$-th diagonal matrix element.

The channel spectral function $B_{a}(M)$ describes the (energy-dependent) component of the full $B(M)$ when projected into a specific interaction channel $a$. It generalizes the prescription of a branching ratio $(B r(X \rightarrow a))$, and incorporates the energy dependence from resonant and non-resonant interactions [3]. The channel yield can thus be readily computed using (2) with $B \rightarrow B_{a}$. A display of the effective spectral functions, in the partial wave (PW) channel: S01, S11, P01, and P11 are shown in Fig. 1.

Few comments are in order:

1. These plots depict in detail how channel opens up above the threshold and the contribution to the overall DoS. For example, S01 is dominated by $\pi \Sigma$ before $\bar{k} N$ opens up. Furthermore, when a specific component dominates a resonance peak it is natural to expect a substantial branching fraction. For example, the first peak in P11 corresponds to a 2 -star $\Sigma(1560)$, predicted to have strong $\pi \Lambda$ and $\pi \Sigma^{*}(1385)$ components. However, branching fractions thus defined are strongly energy dependent, and generally the lineshapes are not well approximated by a sum of Breit-Wigners.

2. The list of identified resonances, even with full details about their widths, is not enough to reconstruct the S-matrix. One way to understand this is to recognize the role of roots in determining the DoS [4]: they encode details of non-resonant interactions.

3. These S-matrix elements are used as input to determine the thermal hyperon yields [8] and to understand LQCD results [6]. For the former, it yields about $\approx 10-30 \%$ increase in the $\Lambda$ yield compared to the standard HRG model. The relatively large uncertainty comes from the poorly constrained unitary backgrounds, which is estimated to make up around $10 \%$ of the total yield at $T=155 \mathrm{MeV}$ (LHC conditions). 

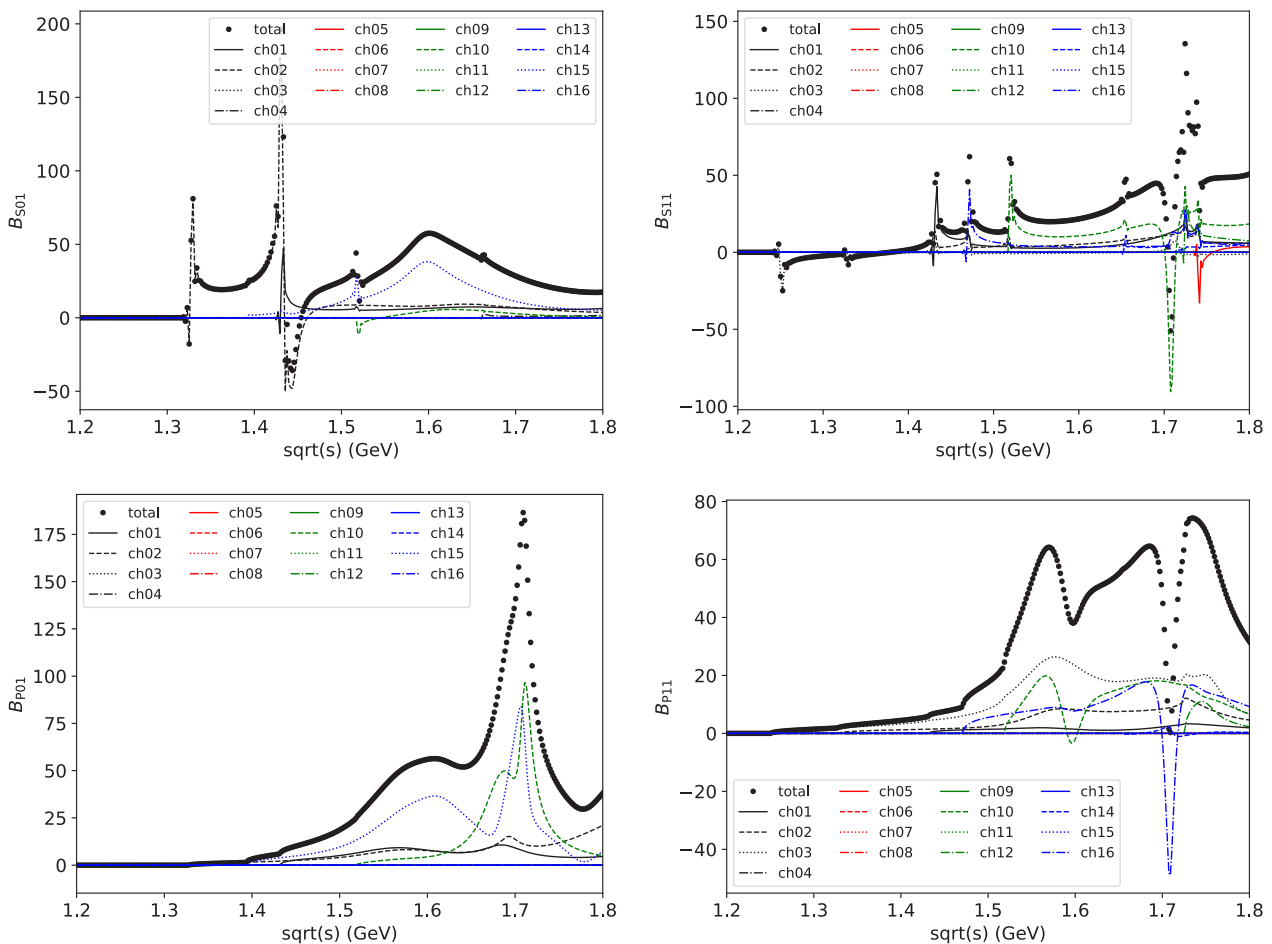

Figure 1. The effective spectral function (with its channel components) as a function of center of mass energy: for S01, S11, P01, and P11 partial waves. See Ref. [8] for details.

\begin{tabular}{|c|c|c|c|c|c|}
\hline channel & elastic & channel & quasi-elastic & channel & unitarity \\
\hline 1 & $\bar{K} N$ & 6 & $\bar{K}_{1}^{*} N$ & 15 & $\pi \pi \Lambda$ \\
\hline 2 & $\pi \Sigma$ & 7 & {$\left[\bar{K}_{3}^{*} N\right]_{-}$} & 16 & $\pi \pi \Sigma$ \\
\hline 3 & $\pi \Lambda$ & 8 & {$\left[\bar{K}_{3}^{*} N\right]_{+}$} & & \\
\hline 4 & $\eta \Lambda$ & 9 & {$[\pi \Sigma(1385)]_{-}$} & & \\
\hline 5 & $\eta \Sigma$ & 10 & {$[\pi \Sigma(1385)]_{+}$} & & \\
\hline & & 11 & {$[\bar{K} \Delta(1232)]_{-}$} & & \\
\hline & & 12 & {$[\bar{K} \Delta(1232)]_{+}$} & & \\
\hline & & 13 & {$[\pi \Lambda(1520)]_{-}$} & & \\
\hline & & 14 & {$[\pi \Lambda(1520)]_{+}$} & & \\
\hline
\end{tabular}

Table 1. The list of interaction channels included in the coupled-channel PWA describing the $|S|=1$ hyperon system by the Joint Physics Analysis Center (JPAC) Collaboration [7]. See Ref. [8] for its thermal model application.

\section{Going further}

The decoupling of dynamics (density of states) and thermo-statistical information is a remarkable feature of the S-matrix formulation of statistical mechanics. In principle, one can make progress in understanding the thermal medium by successively improving the $\mathrm{S}$-matrix 
input: including relevant channels, extension to $\mathrm{N}>2$ scatterings, etc., thus working toward building an accurate virial/cluster expansion.

This is in contrast to the standard imaginary time formulation: interactions (diagrams) are intertwined with temperatures at each step of the Matsubara sum, and one is tasked to compute the in-medium modification of masses and widths. Within a low density expansion $[9,10]$ for some simple cases it is possible to relate the two approaches: the real part of the T-matrix, folded with a thermal distribution integral, provides a shift in the real part of the self-energy; while the imaginary part of T-matrix can be related to resonances $[3,11]$. A more elaborate study of the S-matrix interpretations of the in-medium effects will be reported elsewhere.

\section{4 acknowledgments}

I thank the organizer for the kind invitation to the interesting meeting. I am also grateful to Igor Strakovsky and Christoph Hanhart for the encouraging remarks and many fruitful discussions. This work was supported by the Polish National Science Center (NCN) under the Opus grant no. 2018/31/B/ST2/01663.

\section{References}

[1] R. Dashen, S.K. Ma, H.J. Bernstein, Phys. Rev. 187, 345 (1969)

[2] R. Venugopalan, M. Prakash, Nuclear Physics A 546, 718 (1992)

[3] P.M. Lo, Eur. Phys. J. C 77, 533 (2017), 1707.04490

[4] P.M. Lo, Phys. Rev. D 102, 034038 (2020)

[5] P.M. Lo, B. Friman, K. Redlich, C. Sasaki, Phys. Lett. B 778, 454 (2018), 1710.02711

[6] C. Fernández-Ramírez, P.M. Lo, P. Petreczky, Phys. Rev. C 98, 044910 (2018)

[7] C. Fernandez-Ramirez, I. Danilkin, D. Manley, V. Mathieu, A. Szczepaniak, Phys. Rev. D 93, 034029 (2016), 1510.07065

[8] J. Cleymans, P.M. Lo, K. Redlich, N. Sharma, Phys. Rev. C 103, 014904 (2021), 2009.04844

[9] A. Schenk, Nucl. Phys. B 363, 97 (1991)

[10] A. Schenk, Phys. Rev. D 47, 5138 (1993)

[11] P.M. Lo, F. Giacosa, The European Physical Journal C 79 (2019) 